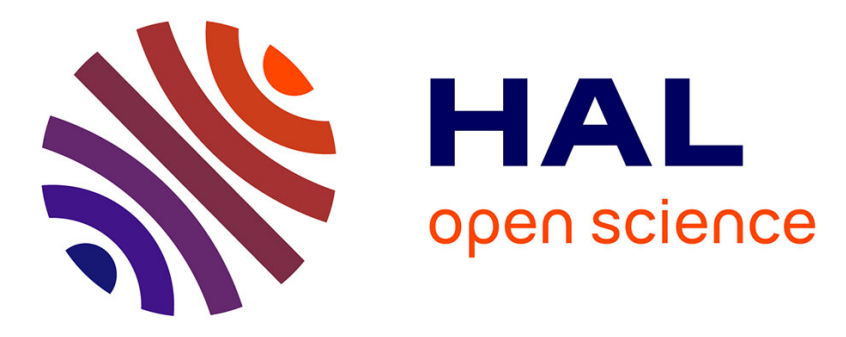

\title{
Adaptive Observer Design under Low Data Rate Transmission with Applications to Oil Well Drill-string
}

Rafael Barreto Jijon, Carlos Canudas de Wit, Silviu-Iulian Niculescu, Jonathan Dumon

\section{- To cite this version:}

Rafael Barreto Jijon, Carlos Canudas de Wit, Silviu-Iulian Niculescu, Jonathan Dumon. Adaptive Observer Design under Low Data Rate Transmission with Applications to Oil Well Drill-string. ACC 2010 - American Control Conference, Jun 2010, Baltimore, Maryland, United States. pp.1973-1978, 10.1109/acc.2010.5531393 . hal-00644154

\section{HAL Id: hal-00644154 \\ https://hal.science/hal-00644154}

Submitted on 23 Nov 2011

HAL is a multi-disciplinary open access archive for the deposit and dissemination of scientific research documents, whether they are published or not. The documents may come from teaching and research institutions in France or abroad, or from public or private research centers.
L'archive ouverte pluridisciplinaire HAL, est destinée au dépôt et à la diffusion de documents scientifiques de niveau recherche, publiés ou non, émanant des établissements d'enseignement et de recherche français ou étrangers, des laboratoires publics ou privés. 


\title{
Adaptive Observer Design under Low Data Rate Transmission with Applications to Oil Well Drill-string
}

\author{
Rafael Barreto Jijón, Carlos Canudas-de-Wit, Silviu-Iulian Niculescu, Jonathan Dumon
}

\begin{abstract}
In oil well drilling operations, one of the important problem to deal with is represented by the necessity of suppressing harmful stick-slip oscillations. A control law named D-OSKIL mechanism uses the weight-on-the-bit force as a control variable to extinguish limit cycles. It uses the value of the bit angular velocity that is found through an unknown parameter observer by means of the measure of the table rotary angular speed. To improve this former estimation, we add the measurement of the angular velocity of the bit that, due to the technological constraints, arrives delayed. This new design leads us to the analysis of a time-varying delay system.
\end{abstract}

Index Terms - Stick-Slip, Oil Well drill string, D-OSKIL, unknown parameter adaptive observer, time-variant, delay, stability.

\section{INTRODUCTION}

Oil well drilling operations present a particular friction phenomenon called Stick-Slip Oscillations which consists in a sub-normal irregular rotation movement, e.g., the top of the drill string rotates with a constant angular velocity, meanwhile downhole, the bit (cutting device) angular velocity varies in a range from zero to six times the one measured at the surface. This effect appears mainly when the bit is in contact with rock formations [1],[10].

Due to the very large forces that are applied into the bit, the presence of stick-slip self-excited oscillations can cause irreversible damage to the equipment, as for example, shut-downs, operation delays, decreasing service life of drill strings and downhole equipment. Since avoiding this kind of oscillations can provide important savings in terms of exploitation time, spare parts costs and maintenance this task has become a challenge for drillers and scientists. Further details about stick-slip and drilling oscillations can be found in [19], [2], [14] and [17].

A model for the drill string system and for the stickslip oscillations as well as an appropriate control law called Drilling-Oscillation Killer (D-OSKIL) have been proposed in [3]. The controller uses mainly a vertical force sometimes named "weight on the bit" (WoB) as an additional variable to eliminate stick-slip effects. Additionally, not all the values of the states of the system are available for control. Adaptive observer has been designed to provide an estimation of such

R. Barreto Jijón is with the INPG, GIPSA-Lab, NeCS team. Grenoble, France. (e-mail: rafael.barreto.jijon@gmail.com)

C. Canudas-de-Wit is with the CNRS, GIPSALab, NeCS team. Grenoble, France. (e-mail: carlos.canudas-de-witegipsa-lab.inpg.fr)

S.-I. Niculescu is with the L2S, CNRS-Supelec, Gif-sur-Yvette, France. (e-mail: Silviu.Niculescullss.supelec.fr)

J. Dumon is with the CNRS, GIPSA-Lab, NeCS team. Grenoble, France. (e-mail: jonathan.dumonegipsa-lab.inpg.fr) states and, additionally, to estimate the friction coefficient. In this case, the measured variable is the rotary angular speed.

Since the physics effects occurring downhole have no strong influence at surface due to the attenuation along the drill string, the measurement does not effectively reflect them. This means that the signal to noise ratio is small and it affects the quality of estimation. We are to improve the observer's behavior, using some coarse information coming from the drilling toll with a new measurement: the bit angular velocity. As explained in the forthcoming section, the technological constraints induce the presence of a transmission delay. It is worth to mention that such a delay introduces an additional difficulty in the control problem as pointed out in the literature in different other cases [8], [16], [13]

The paper is organized as follows: In section II, we briefly introduce the model of the dynamical system along with a description of the testbed where the observer is to be implemented as well as some operational conditions. Section III includes some discussions on the particular physical constraints as well as the technological feasibility of the implementation of the observer. In section IV we tackle the observer's development and its stability conditions and finally, some concluding remarks in section VI end the paper.

Throughout the paper, the following notations will be used: the space $\mathscr{C}_{n, \tau}^{v}$ is a set defined by $\left\{\phi \in \mathscr{C}_{n, \tau}:\|\phi\|_{c}<v\right\}$ where $\mathscr{C}_{n, \tau}=\mathscr{C}\left([-\tau, 0], \mathbb{R}^{n}\right)$ represents the Banach space of the definite piecewise-continuous vector functions mapping the interval $[-\tau, 0]$ into $\mathbb{R}^{n}$ with the uniform convergence topology, and

$$
\|\phi\|_{c}=\sup _{-\tau \leq t \leq 0}\|\phi(t)\|
$$

represents the norm of the function $\phi \in \mathscr{C}_{n, \tau}$.

\section{SYSTEM DESCRIPTION}

\section{A. Model}

The system is modeled as two coupled masses as shown in Figure $1 . J_{r}$ and $J_{b}$ are two inertial masses locally damped by $d_{r}$ and $d_{b}$. The inertias are coupled through an elastic shaft of stiffness $k$ and damping $c$. Let us define $\varphi_{r}, \varphi_{b}$ as the angular positions of the rotary and the bit respectively; $\dot{\varphi}_{r}, \dot{\varphi}_{b}$ as their angular velocities, $u(t)=W o B$ is the weight on the bit control signal, $v(t)$ is the rotary table torque control signal used to regulate $\dot{\varphi}_{r}, \mu$ is the friction coefficient; $A, B, H, C_{o}$ are model matrices given in (3), $\Psi(t)=\Psi(u(t))=H u(t), x$ is the state vector and $y_{o}$ is the output variable. For a more detailed description, see, for instance, [3]. 


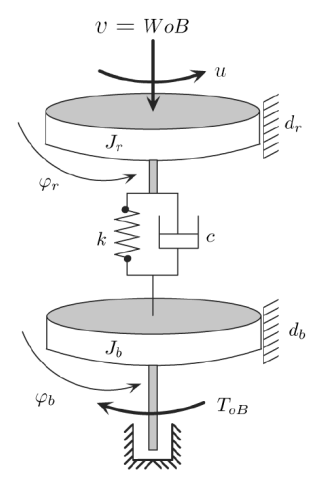

Fig. 1. Drill string two-coupled masses model.

The model to be used is described by:

$$
\begin{aligned}
\dot{x}(t) & =A x(t)+B v(t)+\Psi(t) \mu \\
y_{o} & =C_{o} x=\dot{\varphi}_{r},
\end{aligned}
$$

where the state $x=\left[\begin{array}{lll}x_{1} & x_{2} & x_{3}\end{array}\right]^{T}$ is defined as follows:

$$
x_{1}=\varphi_{r}-\varphi_{b}, \quad x_{2}=\dot{\varphi}_{r}, \quad x_{3}=\dot{\varphi}_{b}
$$

and

$$
\begin{gathered}
A=\left(\begin{array}{ccc}
0 & 1 & -1 \\
-\frac{k}{J_{r}} & -\frac{d_{r}+c}{J_{r}} & \frac{c}{J_{r}} \\
\frac{k}{J_{b}} & \frac{c}{J_{b}} & -\frac{c+d_{b}}{J_{b}}
\end{array}\right), B=\left(\begin{array}{c}
0 \\
\frac{1}{J_{r}} \\
0
\end{array}\right) \\
H=\left(\begin{array}{c}
0 \\
0 \\
-\frac{1}{J_{b}}
\end{array}\right), \quad C_{o}=\left(\begin{array}{lll}
0 & 1 & 0
\end{array}\right)
\end{gathered}
$$

\section{B. Testbed}

In order to validate the proposed results, an appropriate testbed has been built. It is shown in Figure 2 as well as its schematic in Figure 3. It has a Host PC for development, compiling and user interface. A target $\mathrm{PC}$ is also used for real-time execution of the code like the observer and the DOSKIL controller [12]. The rotary system is composed of a DC motor, a transmission box, the rotary table, the drill string, a bit and a quadrature encoder. The support platform consists of a DC motor to move the rotary system vertically, a specimen holder, a support structure and a tension force sensor. The acoustic signal is simulated as sonar pulses like a beeper and microphone pear as shown in Figure 3. Furthermore, the delay is added artificially by software.

We will define the following operation conditions: Well depth: $d \in[0,8000]$ meters, since drilling penetration speed is usually very small, we can consider it as negligible: $\dot{d} \approx 0$. In practice, we have found that $\dot{\varphi}_{b} \in[0,31] \mathrm{rad} / \mathrm{s}$ with typical value $\overline{\dot{\varphi}_{b}}=5 \mathrm{rad} / \mathrm{s}$.

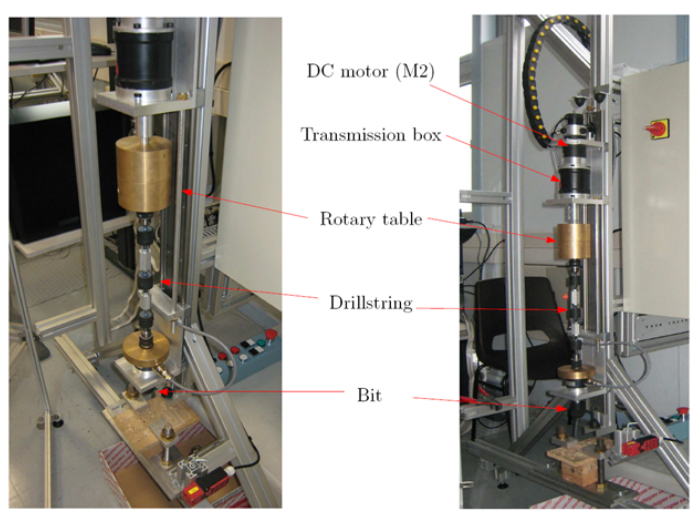

Fig. 2. Experimental setup

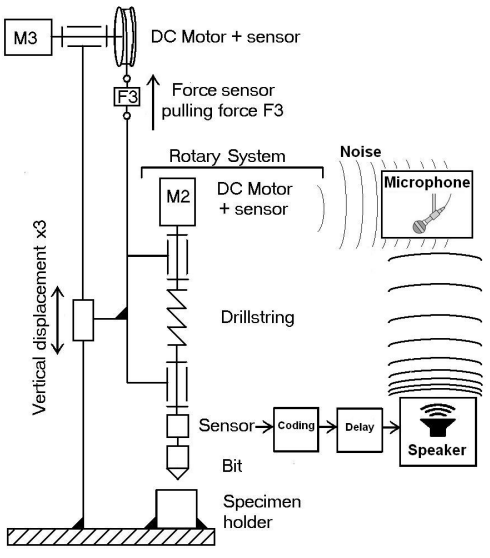

Fig. 3. Testbed schematic with sonar pulses

\section{WIRELESS-TRANSMISSION TECHNOLOGIES}

In this section, we focus on the way to bring the measurement from downhole to surface so we can use it for improving the observer's behavior.

There are mainly two types of transmission: through telemetry signals along the drilling fluid often referred to as mud-pressure pulses [9] and through acoustic waves along the drillstring [4].

In most of the literature, electronic equipments are designed for data acquisition and to play the modulator roll. It should be implemented as an autonomous system energized either by a mud operated electrical turbine or by a battery pack [18].

\section{A. Mud-pulse telemetry}

This technology uses the mud that goes through the drilling system as a transmission media. The data will be represented by pressure pulses. According to [18], the pulser actuator (a stepper-motor-based device) and a main valve restricts the flow and creates some pressure-pulse sequence. A piezoelectric device captures these variations that are then analyzed by a micro-controller. Evidently, due to the irregular nature the mud flow, the low frequency vibrations produced by mud pumps and pulsation dampeners the signals are corrupted by noise. Furthermore, they have an important 
attenuation. Some characteristics to highlight are [11], [4], [7] its cost-effective data transfer, its very low bite rate (1 or 2 bits per second). Mud-pulse velocity declines with the disturbances of mud density, gas content and mud compressibility. It becomes more difficult with increasing well depth. Pulse waves travel through the borehole at 1200 meters per second [11], hence the measure arrives with some delay that increases up to $\tau_{\max } \approx 6.6$ seconds .

\section{B. Acoustic data transmission over a drill string}

Since the acoustic wave propagation velocity in the string material is at least three times superior to that in the mud of the borehole [4], and a higher transmission rate is possible (typically $6 \mathrm{bps}$ ), acoustic transmission seems to be the best way to emit pulses to the surface. These acoustic waves are generated torsional contractions generated by magneto restrictive rings set inside the pipe [6]. In this case $\tau_{\max } \approx 2.2$. It is useful to note that there exists an attenuation of around $4 \mathrm{~dB} / 300 \mathrm{~m}$ [5]. However, we can neglect it because there is always a possibility of setting a repeater at any joint at each 10-15 meters of the section. We consider that this fact does not add any extra considerable delay since the repeater's amplification can occur almost instantaneously.

The telemetry system sends signals directly to the surface through the channel. Usually, there is an embedded sensor measuring $\dot{\varphi}_{b}$ downhole. A measurement noise $S(t)$ is added to the data and then coded all together in such a way it can be transmitted through the acoustic channel $G$. At surface, a receiver will read the encoded signal with the noise $N(t)$. Furthermore, a digital algorithm is used to decode this data and make it available for the use of the observer. Both methods can be modeled by the schematic shown in Figure 4.

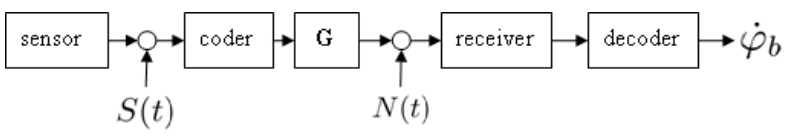

Fig. 4. Block diagram of the transmission channel.

\section{Transmission delay range and friction hypothesis}

Due to technical considerations we can assume that the transmission media is, as a first approximation, like a pure delay system with delay time $\tau \in\left[0, \tau_{\max }\right]$. Moreover, the well's depth increases at a very slow rate and it stops each 1015 meters. In this procedure, the delay can be recalculated. Hence, the delay can be defined as a constant, that is $\dot{\tau}=0$.

On the other hand, we will consider that the friction coefficient is constant or at least slow time variant $\dot{\mu} \approx$ 0 . This approximation is often assumed in the context of adaptive control. This hypothesis means that the rate of variation of the rock friction coefficient does not exhibit substantial changes during drill-operation. Even if the drilled surfaces may have different friction characteristics, the rate of penetration remains small $(\dot{d} \approx 0)$.

\section{ADAPTIVE OBSERVER DESIGN WITH DELAYED FEEDBACK SIGNALS}

A general architecture is proposed in Figure 5 where $\Sigma$ is the system model, $\hat{\Sigma}$ is the observer, $\hat{x}$ is the observed state vector, $K_{o}$ is the default observer gain, $K_{m}$ is the observer gain when the value of $\dot{\varphi}_{b}$ becomes available and $G$ is the transmission channel. Here the outputs of the system are $y_{1}=\dot{\varphi}_{r}$, and $y_{2}=\dot{\varphi}_{b}$.

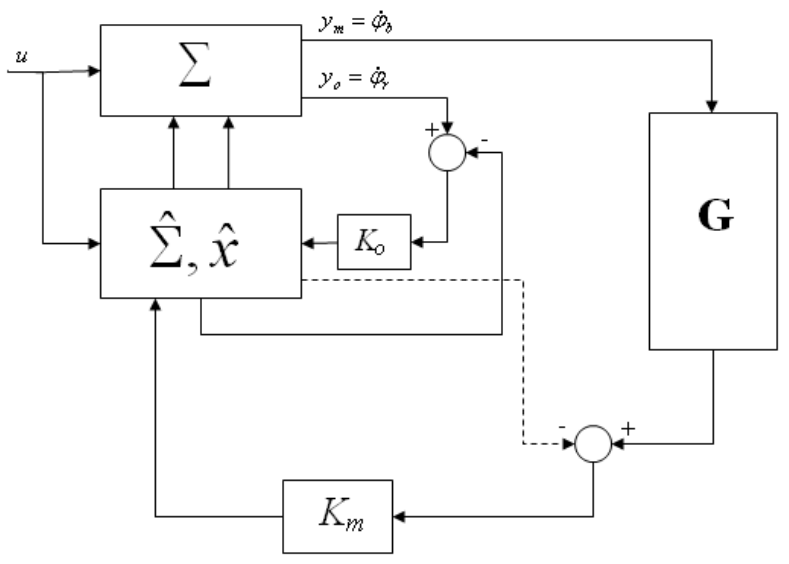

Fig. 5. General Architecture

\section{A. Main Results}

In this section, we will focus on a particular extension of the original observer designed in [3] to handle the delay presence and noise requirements. In this sense, we add two gains related to the delayed measurement as follows:

$$
\begin{aligned}
\dot{\hat{x}}(t)= & A \hat{x}(t)+B v(t)+\Psi(t) \hat{\mu}(t) \\
& +\left[K+\beta \Gamma(t) \Gamma^{T}(t) C_{o}^{T}\right]\left[y_{o}(t)-C_{o} \hat{x}\right] \\
& +K_{x}\left[y_{m}(t-\tau)-C_{m} \hat{x}(t-\tau)\right] \\
\dot{\hat{\mu}}(t)= & \beta \Gamma^{T}(t) C_{o}^{T}\left[y_{o}(t)-C_{o} \hat{x}\right] \\
& +K_{\mu}\left[y_{m}(t-\tau)-C_{m} \hat{x}(t-\tau)\right] \\
\dot{\Gamma}(t)= & \left(A-K C_{o}\right) \Gamma(t)+\Psi(t)
\end{aligned}
$$

where, $y_{m}(t-\tau)$ is the new delayed output vector, $C_{m} \in \mathbb{R}^{1 \times 3}, K_{x} \in \mathbb{R}^{3}$ and $K_{\mu} \in \mathbb{R}$ are the output matrix, and gain matrices for the delayed measurement. $\Gamma(t) \in \mathbb{R}^{3}$ is a matrix generated by the system free of delay (6) such that it generates the states dependent of $\mu$. Here, $\beta$ is a positive scalar and $v(t)$ (and then $\Psi(t)$ ) is persistently exciting, for example $\exists \delta, T>0$ such that the following inequality holds:

$$
\int_{t}^{t+T} \Gamma^{T}(\xi) C_{o}^{T} C_{o} \Gamma(\xi) d \xi>\delta>0
$$

Theorem 1. The unknown parameter adaptive observer described by (4)-(6) guarantees exponential stability for small delays $\tau \in[0, \bar{\tau})$ if there exists some gain matrices $K_{x}$ and $K_{\mu}$ such that

$$
K_{x}(t)=\Gamma(t) K_{\mu}
$$

and the following conditions hold simultaneously: 
(i) $\left(A-K C_{o}\right)$ is Hurwitz, and

(ii) the inequalities

$$
\begin{aligned}
& 0<a_{\text {min }}-K_{\mu} C_{m} \Gamma^{*} \\
& \tau<\bar{\tau}=2 \frac{a_{\text {min }}-K_{\mu} C_{m} \Gamma^{*}}{\left|K_{\mu} C_{m} \Gamma^{*}\right|\left(2\left|K_{\mu} C_{m} \Gamma^{*}\right|+1+a_{\text {max }}^{2}\right)},
\end{aligned}
$$

with:

$$
\begin{aligned}
& a_{\text {min }}=\min _{t \geq 0}\left(\beta \Gamma^{T}(t) C_{o}^{T} C_{o} \Gamma(t)\right), \\
& a_{\text {max }}=\max _{t \geq 0}\left(\beta \Gamma^{T}(t) C_{o}^{T} C_{o} \Gamma(t)\right) .
\end{aligned}
$$

Proof 1. Following the method described in [20] with the constraint (11), we introduce (5) into (4) and then we have the expression of the system (12).

$$
\begin{aligned}
K_{x}(t)= & \Gamma(t) K_{\mu} \\
\dot{\hat{x}}(t)= & A \hat{x}(t)+B v(t)+\Psi(t) \hat{\mu}(t) \\
& +K\left(y_{o}(t)-C_{o} \hat{x}(t)\right)+\Gamma(t) \dot{\hat{\mu}}(t)
\end{aligned}
$$

Let $\tilde{x}(t)=\hat{x}(t)-x(t), \tilde{\mu}(t)=\hat{\mu}(t)-\mu$ with $\dot{\mu}=0$ and since $\dot{\tilde{\mu}}(t)=\dot{\hat{\mu}}(t)-\dot{\mu}$ we obtain:

$$
\dot{\tilde{x}}(t)=\left(A-K C_{o}\right) \tilde{x}(t)+\Psi(t) \dot{\tilde{\mu}}(t)+\Gamma(t) \dot{\tilde{\mu}}(t)
$$

We define the following variable transformation:

$$
\eta(t)=\tilde{x}(t)-\Gamma(t) \tilde{\mu}(t)
$$

then we obtain:

$$
\begin{aligned}
\dot{\eta}(t)= & \left(A-K C_{o}\right)(\eta(t)+\Gamma(t) \tilde{\mu}(t))+\Psi(t) \tilde{\mu}(t)-\dot{\Gamma}(t) \tilde{\mu}(t) \\
= & \left(A-K C_{o}\right) \eta(t) \\
& +\left[\left(A-K C_{o}\right) \Gamma(t)+\Psi(t)-\dot{\Gamma}(t)\right] \tilde{\mu}(t)
\end{aligned}
$$

Due to the system (6), we have:

$$
\dot{\eta}(t)=\left(A-K C_{o}\right) \eta(t)
$$

The system (15) can be assumed as strictly stable e.g. the constant pair $\left(A-K C_{o}\right)$ is detectable. Now, we will focus on analyzing the behavior of $\tilde{\mu}$ :

$$
\begin{aligned}
\dot{\tilde{\mu}}(t) & =\beta \Gamma^{T}(t) C_{o}^{T}\left(y_{o}(t)-C_{o} \hat{x}(t)\right)+K_{\mu} C_{m} \tilde{x}(t-\tau) \\
& =-\beta \Gamma^{T}(t) C_{o}^{T} C_{o} \tilde{x}(t)+K_{\mu} C_{m} \tilde{x}(t-\tau) \\
& =-\beta \Gamma^{T}(t) C_{o}^{T} C_{o}(\eta(t)+\Gamma(t) \tilde{\mu}(t))+K_{\mu} C_{m} \tilde{x}(t-\tau)
\end{aligned}
$$

Since

$$
\tilde{x}(t-\tau)=\eta(t-\tau)+\Gamma(t-\tau) \tilde{\mu}(t-\tau)
$$

then

$$
\begin{aligned}
\dot{\tilde{\mu}}(t)= & -\beta \Gamma^{T}(t) C_{o}^{T} C_{o}(\eta(t)+\Gamma(t) \tilde{\mu}(t)) \\
& +K_{\mu} C_{m}[\eta(t-\tau)+\Gamma(t-\tau) \tilde{\mu}(t-\tau)]
\end{aligned}
$$

Define now:

$$
\begin{aligned}
& a(t)=\beta \Gamma^{T}(t) C_{o}^{T} C_{o} \Gamma(t) \\
& b(t)=-K_{\mu} C_{m} \Gamma(t-\tau)
\end{aligned}
$$

where $0 \leq a(t) \leq \max _{t \geq 0}(a(t))=a_{\max }$ and $b(t)$ are scalars On the other hand, we can approximate

$$
\Gamma^{*}(t)=-\left(A-K C_{o}\right)^{-1} \Psi^{*}(t)=-\left(A-K C_{o}\right)^{-1} H u^{*}(t)
$$

where $\Gamma^{*}, \Psi^{*}$ and $u^{*}$ denote the steady state of $\Gamma(t), \Psi(t)$ and $u(t)$ respectively. Physically, $u(t)$ is a vertical force moving heavy rotating masses with friction. Thus, this movement can not be neither so fast nor sudden. Therefore, we can consider that $u^{*}$ and then $\Gamma^{*}$ vary slowly and they can be treated as constants. Since $\mu$ changes faster than the variability of the gain, we can assume $b(t) \approx-K_{\mu} C_{m} \Gamma^{*} \triangleq b$.

Then

$$
\dot{\tilde{\mu}}(t)=-a(t) \tilde{\mu}(t)-b \tilde{\mu}(t-\tau)+f[\eta(t), \eta(t-\tau)]
$$

Summarizing, we start with the autonomous system $\eta$. We get an adaptation equation by injecting it into that of $\dot{\tilde{\mu}}$. With these "present" and "past" values we compute a estimation of the state vector. The development of the observer's equations are resumed in Figure 6: Since $f[\eta(t), \eta(t-\tau)] \rightarrow 0$ while

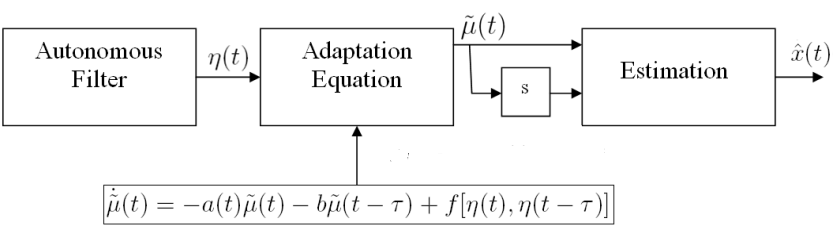

Fig. 6. Observer's equation development Block Diagram

$t \rightarrow \infty$ the problem becomes, at the next step, by analyzing the homogenous part of the equation, to find conditions such that system $\dot{\tilde{\mu}}(t)=-a(t) \tilde{\mu}(t)-b \tilde{\mu}(t-\tau)$ is exponentially stable, and then explicitly compute the gains. For sake of writing simplicity let us define $\chi(t)=\tilde{\mu}(t)$. Then the system becomes:

$$
\dot{\chi}(t)=-a(t) \chi(t)-b \chi(t-\tau)
$$

We have the following result (see the appendix for the proof):

Lemma 2. The time variant delay system described by (20) is asymptotically stable for all $\tau \in[0, \bar{\tau})$ if the following conditions hold:

$$
\begin{aligned}
a_{\text {min }}+b & >0 \\
\bar{\tau} & <2 \frac{a_{\text {min }}+b}{2 b^{2}+\left(a_{\text {max }}^{2}+1\right)|b|}
\end{aligned}
$$

Hence, if there exists $\varepsilon>0, \bar{\tau}>0$ and $a_{\max }>0, a_{\min } \geq 0$ verifying the conditions in Lemma 2 , it is possible to design a gain $b>0$ that makes system (20) asymptotically stable.

Let $\sigma, \varepsilon, \lambda$ three positive scalars. We know that

$$
\begin{aligned}
0 & <\sigma\|\chi(t)\|^{2} \leq V\left(\chi_{t}\right) \\
\dot{V}\left(\chi_{t}\right) & \leq-\varepsilon\|\chi(t)\|^{2} \leq 0,
\end{aligned}
$$

where $V$ represents the Lyapunov-Krasovskii functional allowing us to conclude on the exponential stability of (20):

$$
\frac{\dot{V}\left(\chi_{t}\right)}{V\left(\chi_{t}\right)} \leq-\frac{\varepsilon}{\sigma}
$$


integrating in a positive sense we get:

$$
\int_{0}^{t} \frac{\left.\dot{V}\left(\chi_{\rho}\right)\right)}{\left.V\left(\chi_{\rho}\right)\right)} d \rho \leq \int_{0}^{t}-\frac{\varepsilon}{\sigma} d \rho
$$

Finally, we obtain:

$$
V\left(\chi_{t}\right) \leq \lambda e^{-\frac{\varepsilon}{\sigma} t}
$$

This means that $V\left(\chi_{t}\right)$ decreases exponentially, that implies that the system converges to zero exponentially fast. Thus, if the conditions shown above are held, the system is globally exponentially stable.

This concludes proofs of theorems 1 and 2 .

\section{EXPERIMENTS}

This section reports some preliminary experimental results carried out in the test-bed shown in Fig.2 and Fig.3. The details of this test bed are described in Section II-B. Further details of this experimental test-bed can also be fund in [12].

The experiments correspond to a total run of $77 \mathrm{sec}$ under drilling conditions with constant WoB. These data are collected during the whole run to compute the mean and variance of the estimation error in the two compared cases:

a) Observer without delayed measurements.This corresponds to the case where the gains are set to zero $K_{x}=K_{\mu}=0$

b) Observed using delayed measurements as proposed in this paper with $K_{x}, K_{\mu} \neq 0$.

Table I shown the statistic improvements of our proposed observer over the one that do not use the delayed information.

\begin{tabular}{|c|c|c|}
\hline & mean & variance \\
\hline method $a$ ) & 0.0687 & 2.2308 \\
\hline method $b$ ) & 0.0192 & 0.3382 \\
\hline
\end{tabular}

TABLE I

STATISTICS OF THE OBSERVATIONS ERROR OVER A WHOLE RUN OF 77 SEC.

Figure 7 show the time-history of the bit rotational velocity estimations. The Figures only show a zoom of a period of $1.5 \mathrm{sec}$. The upper figure compares the true measured value of this velocity with the estimated without using the delayed measurements. The lower figure show the improvements that the proposed method.

\section{CONCLUSIONS}

We have discussed the design of an observer for well drilling. We analyzed the system model, the observer's technological feasibility with respect to communication constraints, and operational conditions. Then we designed the general architecture that the new observer should have. We designed an unknown parameter adaptive observer, adding a gain related to a delayed measurement. To prove its exponential stability, we found some simple conditions between the gains of the known and unknown state equations that lead us to a problem of the stability of a linear time-variant
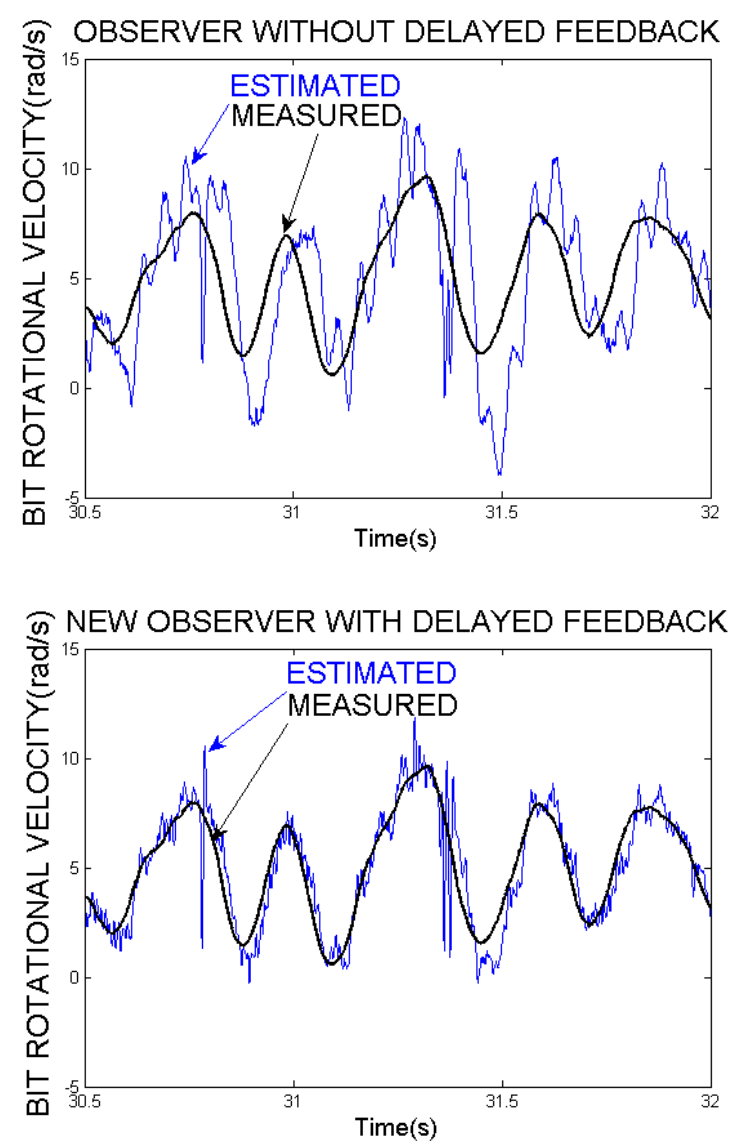

Fig. 7. Time-evolution of the measured bit rotational velocity and the estimation using (lower) and not using (upper) the delayed measurements.

system with delay. We focused in the analysis of such a problem and we found some specific relationships between the former observer's gain and that of the delayed term with respect to the delay time through the Lyapunov's method.

\section{ACKNOWLEDGEMENTS}

This work has been supported by the FeedNetBack European project FP7-ICT-2007-2.

\section{APPENDIX}

Let us rewrite (20) as

$$
\begin{aligned}
\dot{\chi}(t)= & -a(t) \chi(t)-b \chi(t)+b \chi(t)-b \chi(t-\tau) \\
= & -(a(t)+b) \chi(t)+b \int_{-\tau}^{0} \dot{\chi}(t+\theta) d \theta \\
= & -(a(t)+b) \chi(t)-b \int_{-\tau}^{0} a(t+\theta) \chi(t+\theta) d \theta \\
& -b^{2} \int_{-\tau}^{0} \chi(t+\theta-\tau) d \theta
\end{aligned}
$$

Since according to [15], (25) can be viewed as an equation in the $\mathscr{C}_{1,2 \tau}^{v}$ space we have:

$$
\begin{aligned}
\dot{\chi}(t)= & -(a(t)+b) \chi(t)-b \int_{-\tau}^{0} a(t+\theta) \chi(t+\theta) d \theta \\
& -b^{2} \int_{-2 \tau}^{-\tau} \chi(t+\theta) d \theta
\end{aligned}
$$


Let us define a Lyapunov-Krasovskii functional candidate $V\left(\chi_{t}\right)$ of the form

$$
V\left(\chi_{t}\right)=V_{1}(\chi(t))+V_{2}\left(\chi_{t}\right)+V_{3}\left(\chi_{t}\right)
$$

with:

$$
\begin{aligned}
V_{1}(\chi(t)) & =\frac{1}{2} \chi(t)^{2} \geq 0 \\
V_{2}\left(\chi_{t}\right) & =\frac{1}{2} b^{2} \int_{-2 \tau}^{-\tau} \int_{t+\theta}^{t} \chi(\xi)^{2} d \xi d \theta \geq 0 \\
V_{3}\left(\chi_{t}\right) & =\frac{1}{2} a_{\max }^{2}|b| \int_{-\tau}^{0} \int_{t+\theta}^{t} \chi(\xi)^{2} d \xi d \theta \geq 0
\end{aligned}
$$

We take the derivative of $V_{1}(\chi(t))$ along the system (20):

$$
\begin{aligned}
\dot{V}_{1}(\chi(t))= & -(a(t)+b) \chi(t)^{2} \\
& -b \int_{-\tau}^{0} \chi(t) a(t+\theta) \chi(t+\theta) d \theta \\
& -b^{2} \int_{-2 \tau}^{-\tau} \chi(t) \chi(t+\theta) d \theta
\end{aligned}
$$

Since we know that:

$$
\begin{aligned}
-b^{2} \int_{-2 \tau}^{-\tau} \chi(t) \chi(t+\theta) d \theta & \leq \frac{1}{2} b^{2} \tau \chi(t)^{2} \\
& +\frac{1}{2} b^{2} \int_{-2 \tau}^{-\tau} \chi(t+\theta)^{2} d \theta
\end{aligned}
$$

we can rewrite $\dot{V}_{1}$ as:

$$
\begin{aligned}
\dot{V}_{1}(\chi(t)) \leq & -(a(t)+b) \chi(t)^{2} \\
& -b \int_{-\tau}^{0} \chi(t) a(t+\theta) \chi(t+\theta) d \theta+\frac{1}{2} b^{2} \tau \chi(t)^{2} \\
& +\frac{1}{2} b^{2} \int_{-2 \tau}^{-\tau} \chi(t+\theta)^{2} d \theta
\end{aligned}
$$

Let us find the derivative of $V_{2}$ as:

$$
\dot{V}_{2}\left(\chi_{t}\right)=\frac{1}{2} b^{2} \tau \chi(t)^{2}-\frac{1}{2} b^{2} \int_{-2 \tau}^{-\tau} \chi(t+\theta)^{2} d \theta
$$

Finally,

$$
\dot{V}_{3}\left(\chi_{t}\right) \leq \frac{1}{2}|b| a_{\text {max }}^{2} \tau \chi(t)^{2}-\frac{1}{2}|b| a_{\max }^{2} \int_{-\tau}^{0} \chi(t+\theta)^{2} d \theta
$$

Finally, adding all Lyapunov-candidates and using the property that $0 \leq a_{\min } \leq a(t) \leq a_{\max }$, it follows that

$$
\begin{aligned}
\dot{V}(\chi(t)) & =\dot{V}_{1}(\chi(t))+\dot{V}_{2}(\chi(t))+\dot{V}_{3}(\chi(t)) \\
& \leq-\left(a_{\min }+b-b^{2} \tau-\frac{|b| a_{\max }^{2}}{2} \tau-\frac{|b|}{2} \tau\right) \chi(t)^{2} \\
& \leq-\varepsilon \chi(t)^{2}
\end{aligned}
$$

where $\varepsilon>0$.

Hence, according to the Lyapunov-Krasovskii theorem, we need to find a condition for (33) to be negative definite so we can have asymptotic stability for the system (20):

$$
\begin{aligned}
0 & <a_{\text {min }}+b \\
\left(a_{\text {max }}^{2}+1+2|b|\right)|b| \tau & <2\left(a_{\text {min }}+b\right)
\end{aligned}
$$

This concludes the proof.

\section{REFERENCES}

[1] B. Armstrong-Hélouvry. Stick-slip arising from stribeck friction. Proc. IEEE Int. Conf. Robot. Autom., 2:1377-1382, 1990.

[2] A. Baumgart. Stick-slip and bit-bounce of deep-hole drillstrings. $J$. Energy Resour. Technol., 122, 2000.

[3] C. Canudas-de Wit, F. Rubio, and M. A. Corchero. D-OSKIL: A new mechanism for controlling Stick-Slip Oscilations in oil well drillstrings. IEEE Trans. Control Sys. Tech., 16:1177-1191, November 2008.

[4] F. Clayer, H. Heneusse, and J. Sancho. Procède de transmission acoustique de données de forage d'un puits. In World Intellectual Property Organization, March 1992. No. WO 92/04644.

[5] D. Drumheller. Acoustical properties of drill strings. Sandia National Laboratories Research Report, December 1988.

[6] D. Drumheller. An overview of acoustic telemetry. Sandia National Laboratories Research Report, December 1992.

[7] D. Drumheller and S. Knudsen. The propagation of sound waves in drill strings. J. Acoustical Society of America, 97(4):2116-2125, April 1995.

[8] K. Gu, V. L. Kharitonov, and J. Chen. Stability of time-delay systems. Birkhäuser, 2003.

[9] B. Jeffryes, K. Moriarty, and S. Reyes. Method and apparatus for enhanced acoustic mud-pulse telemetry. In World Intellectual Property Organization, September 2001. No. WO 01/66912 A1.

[10] A. Kyllingstad and G. W. Halsey. A study of stick/slip motion of the bit. SPE Drilling Eng., 3-4:369-373, 1988.

[11] X. Liu, B. Li, and Y. Yue. Transmission behavior of mud-pressure pulse along well bore. Journal of Hydrodinamics, 19(2):236-240, 2007.

[12] H. Lu, J. Dumon, and C. Canudas-de Wit. Experimental study of the D-OSKIL mechanism for controlling the stick-slip oscillations in a drilling labratory testbed. IEEE Multiconf. Systems and Control, St Petersburg:Russian Federation, 2009.

[13] W. Michiels and S.-I. Niculescu. Stability and stabilization of timedelay systems. An eigenvalue based approach. SIAM, 2007.

[14] E.M. Navarro-Lopez and Suarez-Cortez. Vibraciones mecánicas en una sarta de perforación: Problemas de control. Revista Iberoamericana de Automática e Informática Industrial, 2:43-54, Jan 2005.

[15] S. Niculescu. Sytèmes à retard. Diderot, 1997.

[16] S.-I. Niculescu. Delay effects on stability. A robust control approach. Springer-Verlag, LNCIS, vol. 269, 2001.

[17] P.D. Spanos, A.M. Chevalier, N.P. Politis, and N.L. Payne. Oil well drilling: A vibrations perspective. Shock Vibration Dig, 35(2):81-99, 2003

[18] P. Tubei, C. Bergeron, and S. Bell. Mud-pulser telemetry system for down hole Measurement-While-Drilling. In IEEE 9th Proc. Instrument and Measurement Tech. Conf., pages 219-223, May 1992.

[19] M. Zamanian, S.E. Kadem, and M.R. Ghazavi. Stick-slip oscillations of drag bits by considering damping of drilling mud and active damping system. J. of Petroleum Science and Eng., 59:289-299, 2007.

[20] Q. Zhang. Adaptive observer for MIMO linear time varying systems. INRIA Rennes Research Report, January 2001. 